\title{
A SURVEY OF WEIGHTED SUBSTITUTION OPERATORS AND GENERALIZATIONS OF BANACH-STONE THEOREM
}

\author{
R. K. SINGH
}

Received 28 April 2004 and in revised form 2 November 2004

The classical Banach-Stone theorem characterizes linear surjective isometries between $C(K)$-spaces. The main aim of this paper is to present a survey of Banach-Stone-theoremtype results between some function spaces. The weighted substitution operators play an important role in characterization of isometries, disjointness preserving operators, and lattice homomorphisms. Some open problems are given for further investigation.

\section{Introduction}

Let $X$ and $Y$ be nonempty sets, let $E$ be a Banach space, and let $B(E)$ denote the Banach space of all bounded linear operators on $E$. Let $F_{1}(X, E)$ and $F_{2}(Y, E)$ be linear spaces of $E$-valued functions on $X$ and $Y$, respectively, with pointwise vector operations. If $\varphi: Y \rightarrow X$ is a map such that the composite function $f \circ \varphi$ belongs to $F_{2}(Y, E)$ for every $f$ in $F_{1}(X, E)$, then the correspondence $S_{\varphi}$ taking $f$ to $f \circ \varphi$ is a linear transformation from $F_{1}(X, E)$ to $F_{2}(Y, E)$ and it is called the substitution transformation (composition transformation) induced by $\varphi$. If $\theta: Y \rightarrow \mathbb{C}$ or $\theta: Y \rightarrow B(E)$ is a map such that $\theta \cdot f \circ \varphi$ belongs to $F_{2}(X, E)$ for every $f$ in $F_{1}(X, E)$, then the mapping $f \rightarrow \theta \cdot f \circ \varphi$ is a linear transformation from $F_{1}(X, E)$ to $F_{2}(Y, E)$ and it is denoted by $W_{\varphi}^{\theta}$. The transformation $W_{\varphi}^{\theta}$ is called the weighted substitution transformation induced by the pair $(\theta, \varphi)$. In case the function spaces $F_{1}(X, E)$ and $F_{2}(Y, E)$ have topologies compatible with the linear structures, and $S_{\varphi}$ and $W_{\varphi}^{\theta}$ are continuous, we call them substitution operator and weighted substitution operator, respectively. Theory of these operators had extensive developments during the last four decades or so and they have been studied on several function spaces of holomorphic functions, continuous functions, and measurable functions and their combinations. For details we refer to $[8,10,11,15,25,28]$.

Let $Y_{1}$ be a subset of $Y, \varphi: Y_{1} \rightarrow X$ and $\theta: Y_{1} \rightarrow \mathbb{C}\left(\right.$ or $B(E)$ ). For $f \in F_{1}(X, E)$, define $\theta \cdot f \circ \varphi$ on $Y$ as $(\theta \cdot f \circ \varphi)(y)=\theta(y) f(\varphi(y))$ for $y$ in $Y_{1}$ and $(\theta \cdot f \circ \varphi)(y)=0$ for $y$ in the complement of $Y_{1}$. In case the correspondence $f \rightarrow \theta \cdot f \circ \varphi$ is continuous, it is called a generalized weighted substitution operator. We also denote it by $W_{\varphi}^{\theta}$.

The weighted substitution operators $W_{\varphi}^{\theta}$ are simple and natural type of operators on function spaces as they come out of two binary operations on functions, namely, 
multiplication and substitution (composition). In some cases, the class of weighted substitution operators contains the class of substitution operators and the class of multiplication operators. Among other applications, these operators have played a significant role in the study of isometries, lattice homomorphisms, and algebra homomorphisms on different spaces of functions. In this paper, we have restricted ourselves to applications of these operators on spaces of continuous functions. These operators have played an important role in generalizations of the classical Banach-Stone theorem in different directions. The paper is expository in nature with some deductions and generalizations. Some problems are presented for further exploration.

\section{Classical Banach-Stone theorem for $C(K)$-spaces}

Let $X$ and $Y$ be compact Hausdorff spaces. Let $E=\mathbb{C}, F_{1}(X, E)=C(X)$ and $F_{1}(Y, E)=$ $C(Y)$, where $C(X)$ and $C(Y)$ are Banach spaces of complex-valued continuous functions on $X$ and $Y$, respectively, with sup norm, that is, $\|f\|=\sup _{x \in X}|f(x)|$. Actually, $C(X)$ and $C(Y)$ are $C^{*}$-algebras with maximal ideal spaces homeomorphic to $X$ and $Y$, respectively. These spaces are called $C(K)$-spaces. In light of the Gelfand-Naimark theorem, we know that every unital commutative $C^{*}$-algebra is a $C(K)$-space. If $X$ is an uncountable metrizable compact space, then in light of Milutin's theorem [23], there exists a linear homeomorphism between $C(X)$ and $C[0,1]$. The classical Banach-Stone theorem characterized linear surjective isometries between $C(K)$-spaces, which turn out to be the weighted substitution operators.

If $\varphi: Y \rightarrow X$ and $\theta: Y \rightarrow \mathbb{C}$ are continuous mappings, then $S_{\varphi}: C(X) \rightarrow C(Y)$ and $W_{\varphi}^{\theta}: C(X) \rightarrow C(Y)$ are continuous operators preserving lattice structures of $C(X)$ and $C(Y) . S_{\varphi}$ is also an algebra homomorphism with $\left\|S_{\varphi}\right\|=1$. In case $\varphi$ is a surjective map, $S_{\varphi}$ is an embedding of $C(X)$ into $C(Y)$ and preserves the norm, that is, $\left\|S_{\varphi} f\right\|=\|f\|$. In case $\varphi$ is a bijection, $S_{\varphi}$ is a surjective isometry. If $|\theta(y)|=1$ for $y \in Y$ and $\varphi$ is a bijection, then it is easy to show that the weighted substitution operator $W_{\varphi}^{\theta}: C(X) \rightarrow C(Y)$ is a surjective isometry. Banach-Stone theorem shows that every surjective isometry is a weighted substitution operator of this type.

Theorem 2.1 (Banach-Stone theorem). Let $X$ and $Y$ be compact Hausdorff spaces. Then a bounded linear operator $T: C(X) \rightarrow C(Y)$ is a surjective isometry if and only if $T=W_{\varphi}^{\theta}$, for some homeomorphism $\varphi: Y \rightarrow X$ and some continuous function $\theta: Y \rightarrow \mathbb{C}$ such that $|\theta(y)|=1$ for every $y \in Y$.

Outline of the proof. Let $M(X)$ and $M(Y)$ denote the Banach spaces of all complexvalued regular Borel measures on $X$ and $Y$, respectively, with total variation norm. The spaces $M(X)$ and $M(Y)$ are the dual spaces of $C(X)$ and $C(Y)$, respectively, and the set of extreme points of the unit ball $(M(X))_{1}$ denoted as $\operatorname{ext}(M(X))_{1}$ coincides with the set $\left\{a \delta_{x}:|a|=1, x \in X\right\}$, where $\delta_{x}: C(X) \rightarrow \mathbb{C}$ is the evaluation functional given by $\delta_{x}(f)=$ $f(x)$ for $f \in C(X)$. If $T: C(X) \rightarrow C(Y)$ is a surjective isometry, then $T^{*}: M(Y) \rightarrow M(X)$ is a surjective isometry and it is a weak ${ }^{*}$-affine homeomorphism of $(M(Y))_{1}$ on $(M(X))_{1}$ such that $T^{*}\left(\operatorname{ext}(M(Y))_{1}\right)=\operatorname{ext}\left((M(X))_{1}\right)$.

Let $y \in Y$. Then $\delta_{y} \in \operatorname{ext}(M(Y))_{1}$. Hence, there exist unique $x \in X$ and $a_{y} \in \mathbb{C}$ such that $\left|a_{y}\right|=1$ and $T^{*}\left(\delta_{y}\right)=a_{y} \delta_{x}$. 
Define $\varphi: Y \rightarrow X$ as $\varphi(y)=x$; and $\theta: Y \rightarrow \mathbb{C}$ as $\theta(y)=a_{y}$. Then $\theta$ is a continuous map and $\varphi$ is a continuous bijection. Since $(T f)(y)=T^{*}\left(\delta_{y}\right)(f)=\theta(y) f(\varphi(y))$ for $y \in Y$, we get $T f=W_{\varphi}^{\theta} f$ for every $f \in C(X)$. This completes the outline of the proof.

Corollary 2.2. (a) If $T: C(X) \rightarrow C(Y)$ is a linear surjective isometry such that $T(1)=1$, then $T=S_{\varphi}$ for some homeomorphism $\varphi: Y \rightarrow X$. (b) If $\varphi: Y \rightarrow X$ is a surjective continuous map, then $S_{\varphi}: C(X) \rightarrow C(Y)$ is an isometry.

Suppose $C(X)$ is the space of all real-valued continuous functions on $X$. Then $C(X)$ is a Banach lattice with $f \vee g$ and $f \wedge g$ defined pointwise, that is, $(f \vee g)(x)=\max \{f(x), g(x)\}$ and $f \wedge g=\min \{f(x), g(x)\}$. A lattice homomorphism between two Banach lattices $E$ and $F$ is a linear mapping $T: E \rightarrow F$ such that $T(f \vee g)=T f \vee T g$ and $T(f \wedge g)=T f \wedge T g$ for every $f, g \in E$. In the following theorem, we find all those linear functionals which are lattice homomorphisms from $C(X)$ to $\mathbb{R}$.

THeOREM 2.3. If $X$ is a compact Hausdorff space and $T: C(X) \rightarrow \mathbb{R}$ is a nonzero continuous linear functional, then $T$ is a lattice homomorphism if and only if $T=c \delta_{x}$ for some $x \in X$ and $c>0$.

Proof. If $T: C(X) \rightarrow \mathbb{R}$ is a nonzero linear functional preserving the lattice structures, then by the Riesz representation theorem, there exists a Borel measure $\mu$ on $X$ such that $T(f)=\int_{X} f d \mu$ for every $f \in C(X)$.

It follows from normality of $X$ that the support of $\mu$ is a singleton set, say $\{x\}$. If $c=\mu(\{x\})$, then $T(f)=\int_{X} f d \mu=\mu(\{x\}) f(x)=c \delta_{x}(f)$ for every $f \in C(X)$. Hence $T=c \delta_{x}$. The converse is easy.

It turns out in light of the above result that every nonzero lattice homomorphism between Banach lattices $C(X)$ and $C(Y)$ is a weighted substitution operator. This is presented in the following theorem.

Theorem 2.4. Let $T: C(X) \rightarrow C(Y)$ be a nonzero positive operator. Then $T$ is a lattice homomorphism if and only if $T=W_{\varphi}^{\theta}$ for some $\theta \in C(Y)$ and for some $\varphi \in C(Y, X)$.

Proof. Let $y \in Y$. Then $\delta_{y} \circ T: C(X) \rightarrow \mathbb{R}$ is a lattice homomorphism. Hence by Theorem 2.3 , there exists a constant $\theta(y) \geq 0$ such that $(T f)(y)=\theta(y) \delta_{x}(f)$ for some $x \in X$. If we define $\varphi(y)=x$, then $\varphi$ is continuous and $T(1)=\theta$. Since $T f=\theta \cdot f \circ \varphi$, we get $T=W_{\varphi}^{\theta}$. If $T$ is a weighted substitution operator, then it is a lattice homomorphism. This completes the proof.

Corollary 2.5. Let $T$ be a positive operator between $C(X)$ and $C(Y)$ such that $T(1)=1$. Then $T$ is a lattice homomorphism if and only if $T$ is a substitution operator if and only if $T$ is an algebra homomorphism.

\section{Characterizations of isometries on some general spaces of continuous functions}

Let $X$ be a locally compact Hausdorff space. Let $V$ be a set of positive real-valued uppersemicontinuous functions on $X$. Then $V$ is said to be a system of weights on $X$ if for $v_{1}, v_{2} \in V$ and $\alpha>0$, there exists $v \in V$ such that $\alpha v_{i} \leq v$ for $i=1,2$ and for every $x \in X$, there exists $v \in V$ such that $v(x)>0$. 
If $V$ is the set of all positive constant functions on $X$ or $V$ is the set of all continuous positive functions vanishing at infinity or $V$ is the set of positive scalar multiples of all characteristic functions, then $V$ is a system of weights on $X$.

If $E$ is a Banach space and $V$ is a system of weights on $X$, then we can define the spaces $C V_{0}(X, E)$ and $C V_{b}(X, E)$ in the following manner:

$$
\begin{aligned}
& C V_{0}(X, E)=\{f \in C(X, E): v f \text { vanishes at infinity for each } v \in V\}, \\
& C V_{b}(X, E)=\{f \in C(X, E): v f(X) \text { is bounded in } E \text { for every } v \in V\} .
\end{aligned}
$$

The spaces $C V_{0}(X, E)$ and $C V_{b}(X, E)$ are linear spaces of continuous $E$-valued functions on $X$. If $v \in V$ and $f \in C(X, E)$, then define $\|f\|_{v}=\sup \{v(x)\|f(x)\|: x \in X\}$. $\|\cdot\|_{v}$ is a seminorm on either $C V_{b}(X, E)$ or $C V_{0}(X, E)$ and the family $\left\{\|\cdot\|_{v}: v \in V\right\}$ of seminorms defines a Hausdorff locally convex topology on each of these spaces. The spaces $C V_{0}(X, E)$ and $C V_{b}(X, E)$ with the locally convex topologies induced by the above family of seminorms are called the weighted spaces of vector-valued continuous functions on $X$. These spaces are natural generalizations of $C(K)$-spaces. For example, if $X$ is compact, then $C V_{0}(X, E)=C V_{b}(X, E)=C(X, E)$. If $V$ is the system of constant weights, then $C V_{0}(X, E)=C_{0}(X, E)$, the space of all continuous functions vanishing at infinity. If $V$ is the set of all positive multiples of characteristic functions, then $C V_{0}(X, E)=C V_{b}(X, E)=$ $C_{0}(X, E)$, the space of all $E$-valued continuous functions with compact supports. For further details about the weighted spaces of continuous functions and some operators on them, we refer to $[22,25,26,30]$. At the place of the Banach space $E$, one can take any locally convex space and define the weighted spaces of vector-valued continuous functions which form a much larger class of locally convex spaces of continuous functions. In this section, we will report Banach-Stone-theorem-type results on some weighted spaces of vector-valued continuous functions.

The first vector-valued generalization of Banach-Stone theorem was obtained by $\mathrm{M}$. Jerison in 1950 in case of strictly convex Banach spaces $E$. Recall that a Banach space $E$ is strictly convex if every vector of unit norm is an extreme point of the closed unit ball of Jerison [13] proved in the following theorem.

Theorem 3.1. Let $X$ and $Y$ be compact Hausdorff spaces and let $E$ be a strictly convex Banach space. Then every linear surjective isometry $T$ between $C(X, E)$ and $C(Y, E)$ is a weighted substitution operator, that is, there exist continuous maps $\varphi: Y \rightarrow X$ and $\theta: Y \rightarrow$ $B(E)$ such that $T=W_{\varphi}^{\theta}$.

In case the dual $E^{*}$ of $E$ is strictly convex instead of $E$, the conclusion of Jerison's theorem holds. This was demonstrated by Lau [17].

If $X$ and $Y$ are locally compact Hausdorff spaces, and $U$ and $V$ are systems of weights of positive constant functions on $X$ and $Y$, respectively, then

$$
C V_{0}(X, E)=C_{0}(X, E), \quad C V_{0}(Y, E)=C_{0}(Y, E) .
$$

For every Banach space $E, C_{0}(X, E)$ and $C_{0}(Y, E)$ are Banach spaces of $E$-valued continuous functions vanishing at infinity on $X$ and $Y$, respectively. The linear isometries between $C_{0}(X, E)$ and $C_{0}(Y, E)$ have been characterized recently in some cases in terms 
of the weighted substitution operators. The following questions are pertinent in this context.

(1) For which Banach spaces $E$ is every surjective linear isometry from $C_{0}(X, E)$ to $C_{0}(Y, E)$ a weighted substitution operator?

(2) In general, for which Banach spaces $E$ and $F$ is every surjective linear isometry from $C_{0}(X, E)$ to $C_{0}(Y, F)$ a weighted substitution operator?

(3) If $C_{0}(X, E)$ is isometrically isomorphic to $C_{0}(Y, E)$, then under what condition are $X$ and $Y$ homeomorphic?

(4) Which isometries from $C V_{0}(X, E)$ to $C V_{0}(X, E)$ are weighted substitution operators? (An operator $T$ from $C V_{0}(X, E)$ to $C V_{0}(X, E)$ is an isometry if $\|T f\|_{v}=$ $\|f\|_{v}$ for $\left.v \in V.\right)$

Some of these questions have already been answered. Classical Banach-Stone theorem and its generalization by Jerison provide some of the answers. Recently Jeang and Wong [12] have provided some answers and examples to some of the questions.

Example 3.2. (a) Let $X=[0,1], Y=\{1\}$, and $Z=\{f \mid f: \mathbb{N} \rightarrow X]$ be the Cartesian product of countably infinite copies of $X$ with product topology. Then $X, Y$, and $Z$ are compact spaces. Let $E=C(Z)$. Then $C(X, E)=C(X, C(Z))=C(X \times Z)=C(Z)$. This is isometrically isomorphic to $C(Y, C(Z))=C(Y, E)$. But $X$ and $Y$ are not homeomorphic. Thus every onto linear isometry may not be a weighted substitution operator.

(b) Let $E=\mathbb{R}_{\infty}^{2}, \mathbb{R} \times \mathbb{R}$ with sup norm. Let $X=\{0,1\}$ and $Y=\{0\}$, with discrete topologies. Define the map $T: C(X, \mathbb{R}) \rightarrow C\left(\{0\}, \mathbb{R}_{\infty}^{2}\right)$ as $T f(0)=(f(0), f(1))$. Then $T$ is a surjective isometry, but it cannot be written as a weighted substitution operator.

If $T$ is a linear map from $C_{0}(X, E)$ to $C_{0}(X, E)$ such that $T$ is a generalized weighted substitution operator $W_{\varphi}^{\theta}$, then both $\varphi$ and $\theta$ are continuous and $\varphi$ and $\theta$ are unique in some sense. This has been demonstrated in [16] and some into isometries are characterized in terms of generalized weighted substitution operators. The following theorem presents this characterization.

Theorem 3.3. If $F$ is a strictly convex Banach space and $E$ is a Banach space, then every linear isometry $T$ between $C_{0}(X, E)$ and $C_{0}(Y, F)$ is a generalized weighted substitution operator.

Outline of the proof. For $\mu \in E^{*}, \mu^{\prime} \in F^{*}$ with $\|\mu\|=\left\|\mu^{\prime}\right\|=1$, and for $x \in X$ and $y \in Y$, define the sets $F_{x}^{\mu}$ and $G_{y}^{\mu^{\prime}}$ as $F_{x}^{\mu}=\left\{f \in C_{0}(X, E): \mu(f(x))=\|f\|=1\right\}$ and $G_{y}^{\mu^{\prime}}=$ $\left\{g \in C_{0}(Y, E): \mu^{\prime}(g(y))=\|g\|=1\right\}$.

If $\delta_{x}$ and $\delta_{y}$ are evaluation functionals at $x$ and $y$, respectively, then $F_{x}^{\mu}$ and $G_{y}^{\mu^{\prime}}$ are norm-attaining sets of the linear functionals $\mu o \delta_{x}$ and $\mu^{\prime} o \delta_{y}$, respectively. If $F_{x}^{\mu}$ is nonempty, then let $Y_{x}^{\mu^{\prime}}=\left\{y \in Y: T\left(F_{x}^{\mu}\right) \subset G_{y}^{\mu^{\prime}}\right.$ for some $\mu^{\prime} \in F^{*}$ with $\left.\left\|\mu^{\prime}\right\|=1\right\}$ and $Y_{x}^{\mu}=\phi$ if $F_{x}^{\mu}=\phi$. It can be shown that $Y_{x}^{\mu} \neq \phi$ if $F_{x}^{\mu} \neq \phi$. Let $Y_{x}$ be the union of $F_{x}^{\mu}$ for $\mu \in E^{*}$ and $\|\mu\|=1$. Then $Y_{x} \neq \phi$ since norm-attaining functionals are dense in the unit sphere of $E^{*}$. Let $Y_{1}$ be the union of all $Y_{x}$ such that $x \in X$.

If $x_{1} \neq x_{2}$, then by strict convexity, $Y_{x_{1}} \cap Y_{x_{2}}=\phi$. Define $\varphi: Y_{1} \rightarrow X$ as $\varphi(y)=x$ if $x \in Y_{x}$. Also $\left(\delta_{y} \circ T\right)(f)=0$ if $\varphi(y)$ does not belong to the support of $f$ for $f \in C_{0}(X, E)$. Thus

$$
\operatorname{ker} \delta_{\varphi(y)} \subset \operatorname{ker} \delta_{y} \circ T \text {. }
$$


Hence there exists a linear map $\theta(y)$ between $E$ and $F$ such that $\delta_{y} \circ T=\theta(y) \delta_{\varphi(y)}$. Equivalently, $T f(y)=\theta(y) f(\varphi(y))$ for $f \in C_{0}(X, E)$ and $y \in Y_{1}$. Thus $T$ is a generalized weighted substitution operator.

Corollary 3.4. If $T: C(X) \rightarrow C(Y)$ is an isometry, then $T$ is a generalized weighted substitution operator. In case $T(1)=1, T$ is a generalized substitution operator.

Note. If $F$ is a real Banach space which is not strictly convex and $E$ is a real Banach space, then a linear isometry has been created in [12] which is not a generalized substitution operator.

If $F$ is a strictly convex Banach space and $x, y \in F$ such that $\|x\|=\|y\|=1$, then $\| x+$ $y \|<2$. This property of $F$ is very significant in characterization of isometries in terms of the weighted substitution operators. If $F$ does not contain a copy of $\mathbb{R}_{2}^{\infty}$, that is, $\mathbb{R}_{2}^{\infty}$ cannot be isometrtically embedded in $F$, then either $\|x+y\|<2$ or $\|x-y\|<2$ for $x, y \in F$ with $\|x\|=1=\|y\|$. It has been shown by Jeang and Wong [12] that if $F$ does not have a copy of $\mathbb{R}_{2}^{\infty}$, then every linear surjective isometry between $C_{0}(X, E)$ and $C_{0}(Y, F)$ is a weighted substitution operator. We will record this in the following theorem.

THeOREM 3.5. If $E$ and $F$ are Banach spaces such that $F$ does not contain a copy of $\mathbb{R}_{2}^{\infty}$, then for every linear surjective isometry between $C_{0}(X, E)$ and $C_{0}(Y, F)$, there exist a continuous $\operatorname{map} \varphi: Y \rightarrow X$ and a continuous map $\theta: Y \rightarrow B(E, F)$ such that $T=W_{\varphi}^{\theta}$ (continuity of $\theta$ is taken with respect to strong operator topology on $B(E, F)$ ).

Let $S_{1}$ be a nonzero closed subspace of $E$. Then $S_{1}$ is said to be an $M$-summand of $E$, if there exists a closed proper subspace $S_{2}$ of $E$ such that

$$
E=S_{1} \oplus S_{2}, \quad\left\|\left(x_{1}, x_{2}\right)\right\|=\max \left\{\left\|x_{1}\right\|,\left\|x_{2}\right\|\right\} .
$$

If $E$ is a reflexive Banach space which does not have any nontrivial direct $M$-summand, then it has been shown by Cambern [4] that every linear surjective isometry from $C_{0}(X, E)$ to $C_{0}(Y, E)$ can be represented as a weighted substitution operator. The problem is still unresolved in case of nonreflexive Banach spaces.

Recall that an operator $T$ from $C V_{0}(X, E)$ to $C V_{0}(X, E)$ is said to be disjointness preserving if $\{x \in X: T f(x) \neq 0\} \cap\{x \in X: \operatorname{Tg}(x) \neq 0\}=\phi$, whenever $\{x \in X: f(x) \neq$ $0\} \cap\{x \in X: g(x) \neq 0\}=\phi$ for $f, g \in C V_{0}(X, E)$. In lattice language, $T$ is disjointness preserving if $f \perp g$ implies that $T f \perp T g$. It turns out that in some cases, the class of disjointness preserving operators contains the class of surjective isometries, that is, every linear surjective isometry on $C_{0}(X, E)$ is a disjointness preserving operator for suitable Banach spaces. In general, if $T$ is a linear surjective isometry such that $T$ and $T^{-1}$ are disjointness preserving, then $T$ can be represented as a weighted substitution operator. We refer to [9] for further details. If $E$ is strictly convex or $E^{*}$ is strictly convex, then every onto isometry is disjointness preserving. The disjointness preserving operators have been studied on the weighted spaces of continuous functions and Banach lattices. For detail, we refer to $[1,2,27]$.

If $V=C_{0}^{+}(X)$, the set of all continuous positive functions vanishing at infinity on $X$, then $C V_{0}(X, E)$ is a seminormed linear space of continuous $E$-valued functions $f$ on $X$ such that $v f$ vanishes at infinity for every $v \in V$. Here each seminorm is a weighted 
analog of sup norm. It has been shown that every continuous disjointness preserving operator $T$ on $C V_{0}(X)$ is a weighted substitution operator, that is, $T=W_{\varphi}^{\theta}$, where $\varphi$ : $X \rightarrow X$ and $\theta: X \rightarrow \mathbb{C}$ such that $\varphi$ is continuous on $\{x \in X: \theta(x) \neq 0)$. A generalization of this result for $C V_{0}(X, E)$ has also been obtained. We present this generalization in the following theorem.

Theorem 3.6. Let $T$ be an operator on $C V_{0}(X, E)$. Then $T$ is disjointness preserving if and only if there exist a function $\varphi: X \rightarrow X$ and a function $\theta \in C V_{0}\left(X, B_{S}(E)\right)$ such that $\varphi$ is continuous on support of $\theta$ and $T=W_{\varphi}^{\theta}$ (here $B_{S}(E)$ denotes the Banach space of all bounded linear operators with strong operator topology).

Outline of the proof. Suppose $T$ is disjointness preserving. Let $t \in E$ and $t^{*} \in E^{*}$ and let $f \in C V_{0}(X)$. Then $f_{t} \in C V_{0}(X, E)$, where $f_{t}(x)=f(x) t$. Define $S_{t}^{t^{*}}$ on $C V_{0}(X)$ as $S_{t}^{t^{*}} f(x)=t^{*}\left(T f_{t}(x)\right)$ for $f \in C V_{0}(X)$ and $x \in X$. Then $S_{t}^{t^{*}}$ is a disjointness preserving operator and hence there exist a self-map $\varphi_{t}^{t^{*}}$, independent of $t$, and $t^{*}$ and a function $\theta_{t}^{t^{*}} \in C V_{0}(X)$ such that $S_{t}^{t^{*}}=W_{\varphi}^{\theta_{t}^{t^{*}}}$, where $\varphi(x)=\varphi_{t}^{t^{*}}(x)$ for all $x \in X$.

For $x \in X$, we define $\theta(x): E \rightarrow E$ as $\theta(x)(t)=\left(t 1_{t}\right)(x)$ for $t \in E$. Then $\theta(x) \in B(E)$ for every $x \in X$ and $\theta \in C V_{0}\left(X, B_{S}(E)\right)$. Now $\theta_{t}^{t^{*}}(x)=S_{t}^{t^{*}} 1(x)=t^{*}\left(T 1_{t}(x)\right)=t^{*}(\theta(x) t)$. From this, we have

$$
t^{*}\left(T f_{t}(x)\right)=S_{t}^{t^{*}} f(x)=t^{*}(\theta(x) t) \cdot f(\varphi(x))=t^{*}\left((\theta)(x) f_{t}(\varphi(x))\right)
$$

for all $f \in C V_{0}(X), t \in E, t^{*} \in E^{*}$, and $x \in\{y \in X: \theta(y) \neq 0\}$. Thus

$$
T f_{t}(x)= \begin{cases}\theta(x)\left(f_{t}(T(x))\right) & \text { if } \theta(x) \neq 0 \\ 0 & \text { otherwise }\end{cases}
$$

Since the set $\left\{f_{t}: f \in C V_{0}(X), t \in E\right\}$ is dense in $C V_{0}(X, E)[22]$, we conclude that $T=$ $W_{\varphi}^{\theta}$. This completes the outline of proof of the theorem.

Note. (1) If $v: X \rightarrow \mathbb{R}^{+}$is a continuous function such that $v(x)>0$ for every $x \in X$, and $V=\{\lambda v: \lambda>0\}$, then $C V_{0}(X, E)$ is a Banach space. It would be nice to characterize linear surjective isometries on $C V_{0}(X, E)$.

(2) Theorem 3.6 is valid even if $E$ is a locally convex Hausdorff space.

(3) Characterization of isometries on general $C V_{0}(X, E)$ is still an open problem and so is the problem of characterization of disjointness preserving operators.

If $E$ is a Banach lattice, then $C_{0}(X, E)$ is also a Banach lattice with order defined as $f \leq g$ whenever $f(x) \leq g(x)$ for every $x \in X$. We have seen in Theorem 2.4 that every nonzero lattice homomorphism between $C(X, \mathbb{R})$ and $C(Y, \mathbb{R})$ is a weighted substitution operator in case $X$ and $Y$ are compact Hausdorff spaces. Actually, $C(X, \mathbb{R})$ is lattice isomorphic to $C(Y, \mathbb{R})$ if and only if $X$ and $Y$ are topologically homeomorphic.

If $E$ and $F$ are Banach lattices, then $C(X, E)$ and $C(X, F)$ may be lattice isomorphic without $X$ and $Y$ being topologically homeomorphic. For example, let $E=\mathbb{R}_{\infty}^{2}$, let $X=$ $\{0\}$, let $Y=\{0,1\}$. Then $T(a, b)=(a, 2 b)$ is a lattice isomorphism from $C\left(X, \mathbb{R}_{\infty}^{2}\right)$ to $C(Y, \mathbb{R})$, but $X$ and $Y$ are not homeomorphic and $\mathbb{R}_{\infty}^{2}$ and $\mathbb{R}$ are not lattice isomorphic. $T$ is also not an isometry. 
If $f \in C(X)$ and $t \in E$, then we know that $f_{t} \in C(X, E)$, where $f_{t}(x)=f(x) t$ for $x \in X$. Let $T: C(X, E) \rightarrow F$ be a lattice homomorphism. Then an element $a \in X$ is a support of $T$ if $T(f)=T\left(1_{f(a)}\right)$ for all $f \in C(X, E)$, where $1 \in C(X)$ is the identity function. If $T: C(X, E) \rightarrow \mathbb{R}$ is a lattice homomorphism such that $T\left(1_{t}\right) \neq 0$, whenever $t \neq 0$, then it has been proved in [5] using Urysohn's lemma that $T$ has a unique support. We will record this in the following theorem which generalizes Theorem 2.3.

Theorem 3.7. Let $T: C(X, E) \rightarrow \mathbb{R}$ be a lattice homomorphism and suppose $T\left(1_{t}\right) \neq 0$ for every $0 \neq t \in E$. Then the support of $T$ is unique.

If $Y$ is a compact Hausdorff space and $F$ is a Banach lattice, then the evaluation map $\delta_{y}$ from $C(Y, F)$ to $F$ is defined as $\delta_{y}(f)=f(y)$. If $T: C(X, E) \rightarrow C(Y, F)$ is a lattice homomorphism, then $\delta_{y} \circ T \rightarrow F$ is a lattice homomorphism. For $y \in Y$, define $T_{y}: E \rightarrow F$ as $T_{y}(t)=\delta_{y} \circ T\left(1_{t}\right)$ for every $t \in E$. The map $\delta_{y} \circ T$ is a lattice homomorphism from $C(X, E)$ to $C(X, \mathbb{R})$ such that $Z(T(f))$ is empty whenever $Z(f)$ is empty, where $Z(f)$ denotes the zero set of $f$. Also $\delta_{y} \circ T\left(1_{t}\right) \neq 0$ when $t \neq 0$. Thus by Theorem 3.7, there exists a unique $x \in X$ such that

$$
\delta_{y} \circ T(f)=\left(\delta_{y} \circ T\right)\left(1_{f(x)}\right)
$$

for every $f \in C(X, E)$. We define the associated map $\varphi: Y \rightarrow X$ as $\varphi(y)=x$ for each $y \in$ $Y$. It turns out that $\varphi$ is a continuous map. If $T: C(X, E) \rightarrow C(Y, \mathbb{R})$ is an onto lattice homomorphism with $Z(T(f))=\phi$, whenever $Z(f)=\phi$, then it is easy to show that $T_{y}$ : $E \rightarrow \mathbb{R}$ is a lattice isomorphism for every $y \in Y$. The following theorem of [5] presents a Banach-Stone-theorem-type result in case of lattice-valued continuous functions on compact Hausdorff spaces $X$ and $Y$.

Theorem 3.8. If $X$ and $Y$ are compact Hausdorff spaces, $E$ is a Banach lattice, and $T$ : $C(X, E) \rightarrow C(Y, \mathbb{R})$ is a lattice isomorphism such that $Z(T(f))$ is empty whenever $Z(f)$ is empty, then $X$ and $Y$ are homeomorphic and $E$ and $\mathbb{R}$ are lattice isomorphic.

Proof. Let $y \in Y$ and $T: C(X, E) \rightarrow C(Y, \mathbb{R})$ be a lattice isomorphism. Then $\delta_{y} \circ T:$ $C(X, E) \rightarrow \mathbb{R}$ is a lattice isomorphism and hence $T_{y}: E \rightarrow \mathbb{R}$ is an isomorphism.

Let $\varphi: Y \rightarrow X$ be the associated map induced by $T$ such that

$$
\delta_{y} \circ T(f)=\delta_{y} \circ T\left(1_{f(y)}\right)
$$

for every $f \in C(X, E)$.

Suppose $x_{0} \in X$ such that $\varphi(y) \neq x_{0}$ for every $y \in Y$. Then there exists an open set $G_{y}$ such that $\varphi(y) \in G_{y}$ and $x_{0} \in \overline{G_{y}}$. The family $\left\{\varphi^{-1}\left(G_{y}\right): y \in Y\right\}$ is an open cover of $Y$. Since $Y$ is compact,

$$
Y=\bigcup_{i=1}^{n} \varphi^{-1}\left(G_{y_{i}}\right) \text { for some finite subset }\left\{y_{1}, y_{2}, \ldots, y_{n}\right\} \text { of } Y
$$

Let $f_{i}$ be a continuous function on $X$ such that $f_{i}\left(x_{0}\right)=1$ and $f_{i}\left(\overline{G_{y_{i}}}\right)=\{0\}$ for $i=$ $1,2, \ldots, n$. If $f=\Lambda_{i=1}^{n} f_{i}$, then $f\left(x_{0}\right)=1$ and $f\left(\overline{G_{y_{i}}}\right)=\{0\}$ for $i=1,2, \ldots, n$. Let $t \in E$ such 
that $t \neq 0$ and let $h=f_{t}$. Then

$$
T(h)(y)=\delta_{y} \circ T(h)=\left(\delta_{y} \circ T\right)(h \circ \varphi(y))=0 .
$$

Thus $T h=0$, and hence $h=0$. But $h\left(x_{0}\right)=f_{t}\left(x_{0}\right)=f\left(x_{0}\right) t=t \neq 0$. This is a contradiction. Hence $\varphi: Y \rightarrow X$ is a surjection. Since $T$ is an isomorphism, $T^{-1}$ is also a lattice isomorphism from $C(Y, \mathbb{R})$ to $C(X, E)$. It can be shown that $Z\left(T^{-1}(g)\right)$ is empty whenever $Z(g)$ is empty. If $\varphi^{\prime}: X \rightarrow Y$ is the associated function corresponding to $T^{-1}$, then it can be shown that $\varphi^{\prime}$ is an inverse of $\varphi$. Hence $\varphi: Y \rightarrow X$ is a homeomorphism, since $X$ and $Y$ are compact spaces. The map $T_{y}: E \rightarrow \mathbb{R}$ is a lattice isomorphism. This completes the proof of the theorem.

Note. It is clear that $C(X, \mathbb{R})$ and $C(Y, \mathbb{R})$ are lattice isomorphic if and only if $X$ and $Y$ are homeomorphic. The substitution operator induced by a homeomorphism from $Y$ to $X$ is a lattice isomorphism from $C(X, \mathbb{R})$ to $C(Y, \mathbb{R})$. As we have seen, $C(X, \mathbb{R})$ and $C(Y, \mathbb{R})$ may be lattice isomorphic without $X$ and $Y$ being homeomorphic. But existence of a lattice isomorphism $T: C(X, E) \rightarrow C(Y, \mathbb{R})$ such that $Z(T(f))$ is empty whenever $Z(f)$ is empty for $f \in C(X, E)$ guarantees that $X$ and $Y$ are homeomorphic. If Banach lattice $E$ with a quasi-interior point has a compact or locally compact representation space [24], then it would be worthwhile to investigate whether a lattice isomorphism from $C(X, E)$ to $C(Y, \mathbb{R})$ gives rise to a homeomorphism from $Y$ to $X$.

\section{Banach-Stone-theorem-type results for algebras}

If $X$ is a completely regular Hausdorff space, then $C(X)$ is an algebra having many continuous functions besides constant functions. In general, if $V$ is a system of weights on $X$, such that $\sqrt{ } v \in V$ whenever $v \in V$ or $V$ contains all constant functions, then $C V_{0}(X)$ is a topological algebra [26]. If $E$ is a Banach algebra, then $C V_{b}(X, E)$ is a topological algebra. If $X$ is a compact Hausdorff space, then $C(X)$ is a $C^{*}$-algebra and every commutative unital $C^{*}$-algebra is isometrically isomorphic to a $C(K)$-space for some compact Hausdorff space $K$. Every linear surjective isometry between $C(K)$-spaces is a weighted substitution operator as we have seen earlier. The concept of a disjointness preserving operator between noncommutative algebras can be discussed in terms of zero-product preserving map which by definition is a linear map $T$ between two algebras $A$ and $B$ such that $T(a) T(b)=0$ whenever $a b=0$. The $C(K)$-spaces carry different mathematical structures on them like commutative ring structure, Banach algebra structure, Banach lattice structure, $C^{*}$-algebra structure, and so forth. It is easy to see in light of results in Section 1 and Section 2 that surjective isometries and lattice isomorphisms between $C(K)$-spaces turn out to be zero product preserving maps. In fact, $T$ preserves zero product between $C(K)$-spaces if and only if $T$ is a weighted substitution operator.

In general if $A$ and $B$ are topological algebras, $T: A \rightarrow B$ is a continuous linear map such that $T(a) T(b)=0$, whenever $a b=0$ and subalgebra generated by the idempotent elements of $A$ is dense in $A$, then $T(e) T(a)=T(a) T(e)$ and $T(e) T(a b)=T(a) T(b)$ for every $a, b \in A$, where $e \in A$ is an identity element. Also $T(a) T(b)=T(b) T(a)$ whenever $a b=b a$. The following theorem of [6] presents a representation of zero-product preserving operators between two topological algebras under certain conditions. 
Theorem 4.1. Let $A$ and $B$ be topological algebras such that $A$ has an identity e, and subalgebra generated by idempotent elements is dense in $A$. Let $T: A \rightarrow B$ be a continuous zeroproduct preserving operator such that $T(e)$ has an inverse in $B$ or the subalgebra generated by $T(A)$ has an identity. Then there exists an algebra homomorphism $S: A \rightarrow B$ such that

$$
T(a)=T(e) S(a) \text { for every } a \in A,
$$

(i.e., $T$ is a weighted algebra homomorphism).

Proof. Suppose $T(e)$ is invertible in $B$. Let $h=T(e)$ and let $S=h^{-1} T$. Then, by remarks given above, it follows that $S$ is an algebra homomorphism and $T=T(e) S$. If $e^{\prime}$ is an identity in subalgebra generated by $T(A)$, then

$$
e^{\prime}=\sum_{i=1}^{k} T\left(a_{i 1}\right) T\left(a_{i 2}\right) \cdots T\left(a_{i k}\right),
$$

where $a_{i k}^{\prime} s$ are elements of $A$. It can be shown that

$$
e^{\prime}=e^{\prime} e^{\prime}=T(e) b=b T(e)
$$

for some $b$ is subalgebra generated by $T(A)$. This shows that $T(e)$ is invertible and hence the proof is completed.

The following corollaries are consequences of this result and its generalization to additive maps preserving zero product on unital rings $A$ generated by idempotents [6].

Corollary 4.2. Let $H$ be an infinite-dimensional Hilbert space and let $B$ be a ring. Let $T: B(H) \rightarrow B$ be a zero-product preserving additive map. Let $B^{\prime}$ be the subring of $B$ generated by $T(B(H))$. Then $T(e)$ belongs to the center of $B^{\prime}$ and

$$
T(e) T(f g)=T(f) T(g), \quad \text { for } f, g \in B(H) .
$$

If $T(e)$ is invertible, then $T=T(e) S$ for some ring homomorphism $S$ from $B(H)$ to $B$.

Corollary 4.3. Let $H_{1}$ and $H_{2}$ be infinite-dimensional Hilbert spaces. If $T: B\left(H_{1}\right) \rightarrow$ $B\left(H_{2}\right)$ is an additive bijection preserving zero product, then

$$
T(f)=\alpha g^{-1} f g \quad \text { for } f \in B\left(H_{1}\right),
$$

where $\alpha$ is a scalar and $g$ is an invertible operator from $H_{2}$ to $H_{1}$, that is, $T=W_{g}^{\alpha g^{-1}}$.

Note. Some results similar to Theorem 4.1 and its corollaries have been obtained for operator algebras, $W^{*}$-algebras and $C^{*}$-algebras. We refer to $[3,6,7]$ for details.

The characterizations of isometrics between function spaces and function algebras of integrable functions and analytic functions have also been obtained in terms of the weighted substitution operators. In 1958, Lamperti [16] proved that every surjective linear isometry on $L^{p}(X, \mu)(p \neq 2)$ is a weighted substitution operator and this result was extended in 1978 by Sourour [29] to some $L^{p}$-spaces of vector-valued functions. Linear surjective isometries on Hardy spaces $H^{p}(D), p \neq 2$, were characterized in terms of 
the weighted substitution operators and were extended to Hardy spaces of some vectorvalued analytic functions by Lin and Prolla $[18,22]$. Pathak $[20,21]$ studied isometries on spaces of absolutely continuous functions and on $C^{n}[0,1]$.

The isometries of operator algebras and function algebras were also studied. Kadison [14] initiated the work on isometries of operator algebras. Recently Blecher and Labuschagne [3] have made further investigations regarding isometries on operator algebras. Matheson [19] has characterized linear isometries between uniform algebras in terms of (generalized) weighted substitution operators. Isometries between nice function spaces are the weighted substitution operators. But we have seen examples of isometries and lattice homomorphisms which cannot be represented as the weighted substitution operators. In characterization of isometries between function spaces, a three-layer mathematical structure is employed, namely, underlying spaces, function spaces over underlying spaces, and dual spaces of function spaces. In most of the cases, evaluation functionals play important role in obtaining a map between underlying spaces giving rise to substitution operator between function spaces. In case of the Banach algebras of functions, the maximal ideal space plays a crucial part. In case it is complicated, the representation of isometric isomorphisms becomes difficult. The surjective isometries, lattice homomorphisms, and disjointness preserving operators between function spaces are important transformations preserving some mathematical structures and it is nice to know that in most of the cases they evolve from two basic binary operations on functions, namely, multiplication and composition justifying the significance of the study of the weighted substitution operators on function spaces.

\section{Acknowledgments}

The work on this exposition was initiated during the summer of 2003 when the author visited the University of Massachusetts at Boston and the University of Texas at Austin. The author is thankful to Professors Herbert Kamowitz and Dennis Wortman of Boston and to Professor Haskell Rosenthal of Austin for their invitations and interactions. The author is grateful to the referees of this paper for their constructive critical comments and suggestions.

\section{References}

[1] Y. A. Abramovich, Multiplicative representation of disjointness preserving operators, Nederl. Akad. Wetensch. Indag. Math. 45 (1983), no. 3, 265-279.

[2] Y. A. Abramovich and A. K. Kitover, A solution to a problem on invertible disjointness preserving operators, Proc. Amer. Math. Soc. 126 (1998), no. 5, 1501-1505.

[3] D. P. Blecher and L. E. Labuschagne, Logmodularity and isometries of operator algebras, Trans. Amer. Math. Soc. 355 (2003), no. 4, 1621-1646.

[4] M. Cambern, A generalized Banach-Stone theorem, Proc. Amer. Math. Soc. 17 (1966), 396-400.

[5] J. Cao, I. Reilly, and H. Xiong, A lattice-valued Banach-Stone theorem, Acta Math. Hungar. 98 (2003), no. 1-2, 103-110.

[6] M. A. Chebotar, W.-F. Ke, P.-H. Lee, and N.-C. Wong, Mappings preserving zero products, Studia Math. 155 (2003), no. 1, 77-94.

[7] C.-H. Chu and N.-C. Wong, Isometries between $C^{*}$-algebras, Rev. Mat. Iberoamericana 20 (2004), no. 1, 87-105. 
[8] W. Feldman, Compact weighted composition operators on Banach lattices, Proc. Amer. Math. Soc. 108 (1990), no. 1, 95-99.

[9] S. Hernandez, E. Beckenstein, and L. Narici, Banach-Stone theorems and separating maps, Manuscripta Math. 86 (1995), no. 4, 409-416.

[10] J. E. Jamison and M. Rajagopalan, Weighted composition operator on $C(X, E)$, J. Operator Theory 19 (1988), no. 2, 307-317.

[11] J.-S. Jeang and N.-C. Wong, Weighted composition operators of $C_{0}(X)$ 's, J. Math. Anal. Appl. 201 (1996), no. 3, 981-993.

[12] On the Banach-Stone problem, Studia Math. 155 (2003), no. 2, 95-105.

[13] M. Jerison, The space of bounded maps into a Banach space, Ann. of Math. 60 (1975), $229-233$.

[14] R. V. Kadison, Isometries of operator algebras, Ann. of Math. (2) 54 (1951), 325-338.

[15] H. Kamowitz, Compact weighted endomorphisms of $C(X)$, Proc. Amer. Math. Soc. 83 (1981), no. 3, 517-521.

[16] J. Lamperti, On the isometries of certain function-spaces, Pacific J. Math. 8 (1958), 459-466.

[17] K. S. Lau, A representation theorem for isometries of $C(X, E)$, Pacific J. Math. 60 (1975), no. 1, 229-233.

[18] P.-K. Lin, The isometries of $H^{\infty}(E)$, Pacific J. Math. 143 (1990), no. 1, 69-77.

[19] A. Matheson, Isometries into function algebras, Houston J. Math. 30 (2004), no. 1, 219-230.

[20] V. D. Pathak, Isometries of $C^{n}[0,1]$, Pacific J. Math. 94 (1981), no. 1, 211-222.

[21] Linear isometries of spaces of absolutely continuous functions, Canad. J. Math. 34 (1982), no. 2, 298-306.

[22] J. B. Prolla, Weighted approximation of continuous functions, Bull. Amer. Math. Soc. 77 (1971), 1021-1024.

[23] H. P. Rosenthal, The Banach spaces $C(K)$, Handbook of the Geometry of Banach Spaces, Vol. 2, North-Holland, Amsterdam, 2003, pp. 1547-1602.

[24] H. H. Schaefer, Banach Lattices and Positive Operators, Springer, New York, 1974.

[25] R. K. Singh and J. S. Manhas, Composition Operators on Function Spaces, North-Holland Mathematics Studies, vol. 179, North-Holland Publishing, Amsterdam, 1993.

[26] - Weighted topological algebras of vector-valued functions, J. Indian Math. Soc. (N.S.) 60 (1994), no. 1-4, 59-69.

[27] R. K. Singh and B. Singh, Lamperti-type operators on a weighted space of continuous functions, Proc. Amer. Math. Soc. 125 (1997), no. 4, 1161-1165.

[28] R. K. Singh and W. H. Summers, Compact and weakly compact composition operators on spaces of vector valued continuous functions, Proc. Amer. Math. Soc. 99 (1987), no. 4, 667-670.

[29] A. R. Sourour, The isometries of $L^{p}(\Omega, X)$, J. Funct. Anal. 30 (1978), no. 2, 276-285.

[30] W. H. Summers, Weighted locally convex spaces of continuous functions, Ph.D. thesis, Louisiana State University, Louisiana, 1968.

R. K. Singh: Department of Mathematics, Faculty of Sciences, University of Jammu, Jammu180006, India

E-mail address: rajkishor@hotmail.com 


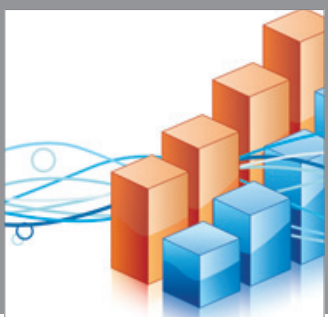

Advances in

Operations Research

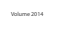

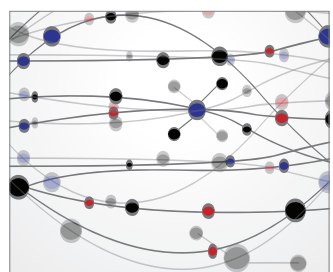

\section{The Scientific} World Journal
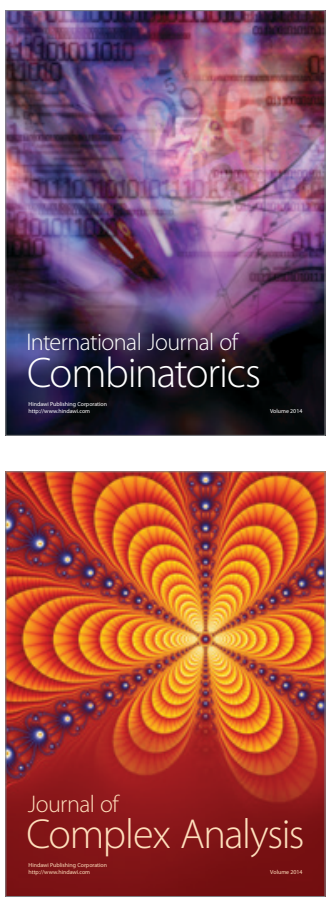

International Journal of

Mathematics and

Mathematical

Sciences
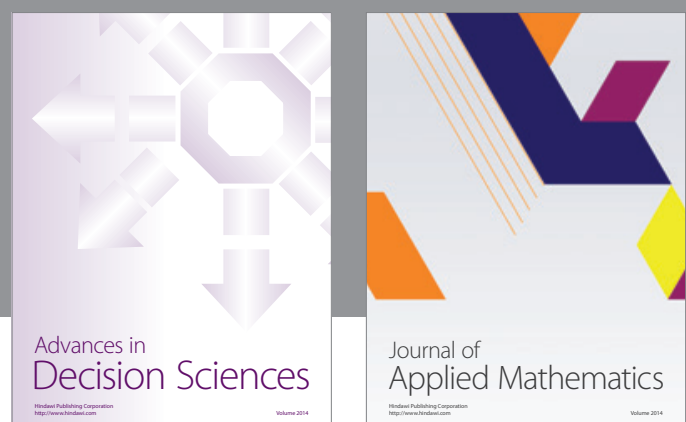

Journal of

Applied Mathematics
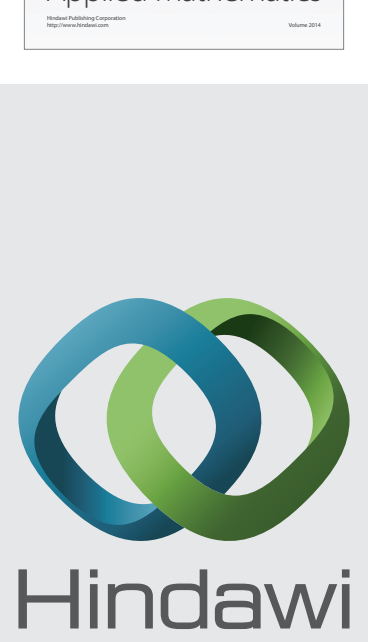

Submit your manuscripts at http://www.hindawi.com
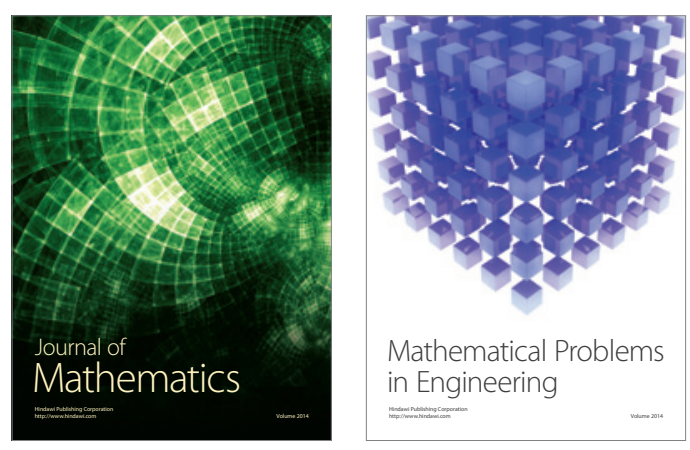

Mathematical Problems in Engineering
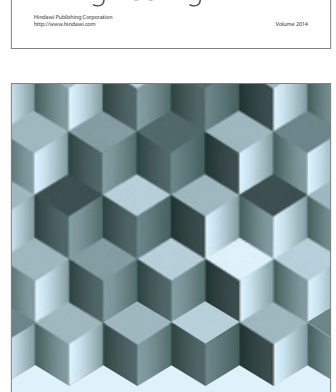

Journal of

Function Spaces
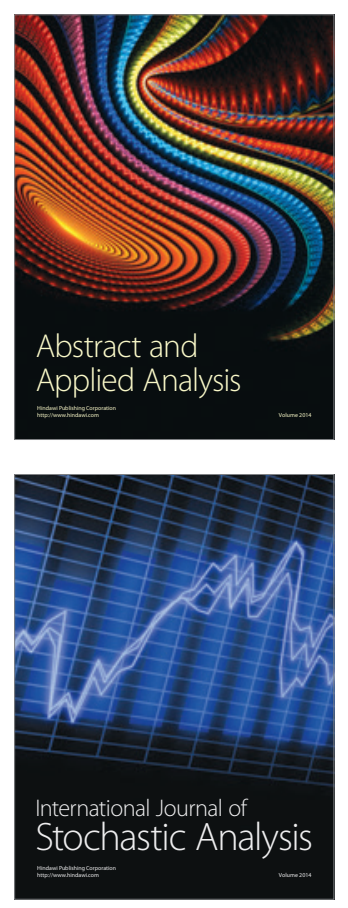

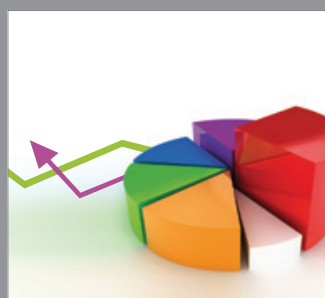

ournal of

Probability and Statistics

Promensencen
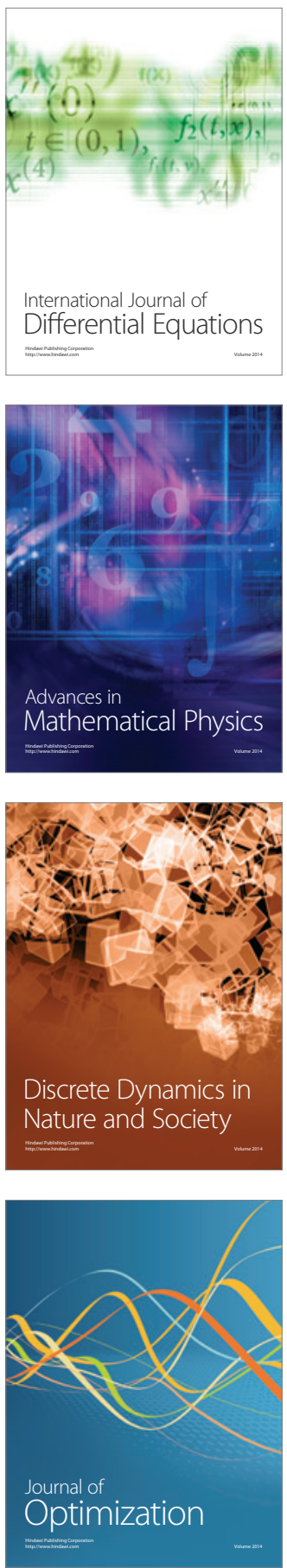